\title{
Daratumumab for Immunoglobulin Light Chain Amyloidosis
}

\author{
Foteini Theodorakakou, Meletios A Dimopoulos and Efstathios Kastritis \\ Department of Clinical Therapeutics, National and Kapodistrian University of Athens, School of Medicine, Athens, Greece
}

DOI: https://doi.org/10.17925/OHR.2021.17.2.79

mmunoglobulin light chain (AL) amyloidosis is characterized by small, indolent plasma cell clones that uniformly demonstrate high expression of CD38 surface molecule and produce toxic, free light chains. The use of daratumumab, a human anti-CD38 monoclonal

antibody, has shown important efficacy with minor toxicities in patients with relapsed or refractory AL amyloidosis. Early data from the phase III ANDROMEDA clinical trial, which evaluated the combination of subcutaneous daratumumab with standard-of-care cyclophosphamide, bortezomib and dexamethasone in patients with newly diagnosed AL amyloidosis, showed an outstanding rate of deep haematological response with significant improvement in organ function, results that led to the approval of daratumumab for the treatment of the disease. A new era for the management of AL has arisen and daratumumab is the centrepiece. In the current article, we provide an overview of the mechanism of action of daratumumab and review the clinical evidence that supports its use in the management of AL amyloidosis.

\section{Keywords}

Light chain amyloidosis, monoclonal antibody, daratumumab

Disclosures: Efstathios Kastritis has received honoraria/ personal fees from Amgen, Genesis Pharma, Janssen, Takeda and Prothena, and research grants from Amgen and Janssen. Meletios A Dimopoulos has received honoraria/personal fees from Amgen, BMS, Celgene, GSK, Janssen, Takeda. Foteini Theodorakakou has no financial or non-financial relationships or activities to declare in relation to this article.

Review process: Double-blind peer review.

Compliance with ethics: This article involves a review of the literature and did not involve any studies with human or animal subjects performed by any of the authors.

Data availability: Data sharing is not applicable to this article as no datasets were generated or analysed during the writing of this article.

Authorship: The named authors meet the Internationa Committee of Medical Journal Editors (ICMJE) criteria for authorship of this manuscript, take responsibility for the integrity of the work as a whole, and have given final approval for the version to be published. Access: This article is freely accessible at touchONCOLOGY.com (c) Touch Medical Media 2021.

Received: 15 September 2021

Accepted: 20 November 2021

Published online: 14 December 2021

Citation: touchREVIEWS in Oncology \& Haematology. 2021;17(2):79-83

Corresponding author: Efstathios Kastritis, Department of Clinical Therapeutics, National and Kapodistrian University of Athens, School of Medicine, Athens, Greece E: ekastritis@gmail.com; ekastritis@med.uoa.gr

Support: No funding was received in the publication of this article.
Immunoglobulin light chain (AL) amyloidosis is the most common type of systemic amyloidosis, a spectrum of diseases induced by misfolded proteins that generate insoluble amyloid fibrils and deposits in various organs. The precursor protein in AL amyloidosis is a toxic, free light chain (FLC) secreted by an indolent plasma cell or B-cell clone in the bone marrow. ${ }^{1}$ The most affected organs include the kidney, heart, liver, peripheral and autonomous nervous systems, and soft tissues. Heart involvement drives down survival of patients, according to the Mayo risk stratification system, which is based on cardiac biomarkers (N-terminal of the prohormone brain natriuretic peptide and high-sensitivity cardiac troponin $\mathrm{T}$ ) and revised by the incorporation of difference of serum free light chain (dFLC).,2,3

Treatment of AL amyloidosis is aimed at eradication of the underlying clone. The backbone of therapy includes regimens adopted from the field of multiple myeloma (MM). These anticlonal agents include alkylators (melphalan and cyclophosphamide), proteasome inhibitors (bortezomib, carfilzomib and ixazomib) and immunomodulatory drugs (lenalidomide and pomalidomide). Early recognition and deep haematological responses, defined as very good partial response (VGPR) or better, have been correlated with improved overall survival (OS) over recent decades, with 2-year OS of $60 \%$ and 4 -year OS of $54 \%{ }^{4}$ However, the disease is still incurable and $30 \%$ of patients will still die within 6 months of diagnosis, while a significant number are prone to toxicities related to conventional treatments. The development of more effective combinations that will further improve haematological responses will lead to prolonged survival and organ function restoration. ${ }^{5}$ Recently, the results of the phase III, randomized, open-label, multicentre ANDROMEDA (AMY3001) trial have been published. ${ }^{6}$ The study evaluated the combination of subcutaneous (SC) daratumumab with standard-of-care cyclophosphamide, bortezomib and dexamethasone (CyBorD) in newly diagnosed AL amyloidosis. ${ }^{6}$ Based on ANDROMEDA, the US Food and Drug Administration (FDA) and European Medicines Agency approved the use of SC daratumumab in combination with CyBorD for the treatment of newly diagnosed AL amyloidosis with Mayo stage I-IIIA, making it the first approved therapeutic regimen for this unique disease?

This article provides an overview of the mechanism of action of daratumumab, reviews the clinical evidence that supports its use in AL amyloidosis and describes how daratumumab could be incorporated into the current treatment strategy.

\section{CD38 in AL amyloidosis clone}

The biology of the plasma cell clone is considered to play a significant role in the pathogenesis of AL amyloidosis. The bone marrow usually shows low-grade plasma cell clones with low proliferation index. ${ }^{8}$ In fact, the presence of bone marrow plasma cells $>10 \%$ is associated with poor survival regardless of Mayo stage. ${ }^{9}$ Clonal plasma cells produce large amounts of amyloidogenic FLCs that increase oxidative stress and render plasma cells highly dependent on proteasome activity. Thus, amyloidotic cells are intrinsically sensitized to proteasome inhibitors and bortezomib has become the centrepiece in AL amyloidosis management. ${ }^{10}$ Whole-genome sequencing studies 
reveal that $\mathrm{AL}$ amyloidosis clones have more features in common with monoclonal gammopathy of undetermined significance than with $\mathrm{MM}^{11}$ while the most frequent chromosomal aberration is translocation (11;14), which is also associated with adverse outcomes in patients treated with bortezomib-based regimens. ${ }^{12}$

Studies using flow cytometry and next-generation sequencing have demonstrated that most clonal plasma cells express CD38, while higher expression has been correlated with advanced cardiac involvement and adverse prognosis in AL amyloidosis. ${ }^{13,14}$ CD38 is a $45-K D$, type 2 transmembrane molecule with dual function both as transmembrane signalling receptor and as enzyme. Interaction with its ligand, CD31, leads to cell survival and migration. ${ }^{15}$ Moreover, CD38 acts as an ectoenzyme by converting nicotinamide adenine dinucleotide to cyclic adenosine diphosphate-ribose, which regulates calcium release and lymphocyte proliferation. ${ }^{16} \mathrm{CD} 38$ is present on both normal and malignant plasma cells, but also on regulatory B cells, regulatory T cells and natural killer cells. ${ }^{17-19}$ The rationale for targeting the CD38 molecule in myeloma and other plasma cell dyscrasias lies in the fact that CD38 is ubiquitously expressed on plasma cells and on its role in cell signalling.

\section{Daratumumab mechanism of action}

The small size and the indolent nature of the AL amyloidosis plasma cell clone make therapy with monoclonal antibodies feasible and optimal. Daratumumab is a human IgGK1 monoclonal antibody (MoAb) that binds with high affinity to CD38 on clonal plasma cells and other lymphoid cells. Similarly to antibodies, MoAbs have two distinct functional domains: a constant fragment $(\mathrm{FC})$ that connects to effector cells via FCy receptors $(\mathrm{F} C \gamma \mathrm{R})$ and a variable fragment that binds to antigens. Daratumumab mediates cytotoxicity of plasma cells through pleiotropic mechanisms, including antibody-dependent cellular cytotoxicity (ADCC), antibodydependent cellular phagocytosis (ADCP), complement-dependent cytotoxicity and direct apoptosis. ${ }^{20}$ Binding of the Fc domain to the CD38 epitope on clonal plasma cells promotes the activation of natural killer cells, which results in degranulation of cytotoxic cytokines and lysis of tumour cells by $A D C C_{1}{ }^{21}$ and the activation of other effector cells (macrophages and monocytes), which leads to phagocytosis (ADCP). ${ }^{22}$ Similarly, the interaction of daratumumab with C1q activates the classical complement pathway and the formation of membrane attack complex. ${ }^{20}$ Daratumumab induces programmed cell death of CD38+ myeloma cells through FcyR-mediated cross-linking. ${ }^{23} \mathrm{CD} 38$ redistribution on the surface, loss of membrane integrity and morphological modifications contribute to programmed cell death activation. ${ }^{23}$ Daratumumab has immunomodulatory effects, too. ${ }^{24}$ The absolute number of natural killer cells is significantly reduced during daratumumab therapy. ${ }^{25}$ Regulatory T cells and other myeloid-derived suppressor cells are also depleted. ${ }^{18}$ At the same time, a substantial increase in CD4+ Th1 cells and CD8+ effector $T$ cells could enhance an adaptive immune response. ${ }^{18}$ Finally, changes in enzymatic activity of daratumumab have also been studied. ${ }^{26}$ Inhibition of CD38 ectoenzyme function decreases the release of adenosine, an immunosuppressor that eliminates the activity of immune effector cells. ${ }^{21}$

\section{Daratumumab in AL amyloidosis}

Daratumumab monotherapy has produced durable responses with a manageable toxicity profile in relapsed/refractory MM. ${ }^{27,28}$ The addition of daratumumab to lenalidomide or bortezomib significantly prolonged progression-free survival (PFS) and improved the overall response rate of patients who had received at least one prior treatment.29,30 Among patients with newly diagnosed $\mathrm{MM}$, both eligible and ineligible for autologous stem cell transplantation (ASCT), daratumumab in combination with standard-of-care regimens improved OS and PFS. ${ }^{31-34}$ Furthermore, patients achieved deeper responses, and evaluation of minimal residual disease (MRD) showed higher MRD-negative rates. ${ }^{32-36}$ The results of the COLUMBA study showed that SC daratumumab $(1,800 \mathrm{mg}$ in $15 \mathrm{~mL})$ with recombinant human hyaluronidase $\mathrm{PH} 20$, was non-inferior to intravenous (IV) daratumumab in terms of efficacy and pharmacokinetics, and led to FDA approval. The most common side effects of daratumumab are infusion-related reactions and infections.

In AL amyloidosis, daratumumab was initially introduced in the relapsed setting. The first report was published in 2016. Two patients with previously treated AL amyloidosis received daratumumab with dose and schedule similar to those used for myeloma approval. Both patients achieved haematological complete remission soon after they began treatment. ${ }^{37}$ One of the patients had cardiac involvement and tolerated the IV infusion of daratumumab, suggesting that the treatment may also be an option for frail patients. A retrospective analysis of 25 heavily pretreated patients, published in 2017 by Kaufman et al. showed that daratumumab produced rapid and deep responses. ${ }^{38}$ The overall haematological response rate was $76 \%$ (complete response [CR] in 36\% and VGPR in $24 \%$ ), and median time to response was 1 month. Responses were actually more favourable than those with other available regimens in the relapse setting, and adverse events were similar to those observed in myeloma. ${ }^{38}$ Further small cohorts of patients with relapsed AL amyloidosis who received IV daratumumab had extraordinary haematological responses (63-100\%), time to response (1-4 weeks) and organ response (cardiac response in up to $55 \%$ and renal response in up to $67 \%$ ). ${ }^{39-42} \mathrm{~A}$ large cohort of patients $(n=168)$ compared daratumumab monotherapy with the combination of daratumumab plus bortezomib and dexamethasone. ${ }^{43}$ Results were promising, with $64 \%$ versus $66 \%$ of patients achieving $C R$, showing that combination therapies are feasible in relapsed $\mathrm{AL}$ amyloidosis. Daratumumab has been an effective option even for patients with high tumour burden or nephrotic-range proteinuria..$^{44}$ Moreover, daratumumab in combination with immunomodulatory drugs appears to be safe and more effective than in MM patients. ${ }^{45}$ These results provided the rationale for the ANDROMEDA study in newly diagnosed patients. ${ }^{6.46}$

\section{Daratumumab in combination with cyclophosphamide, bortezomib and dexamethasone in newly diagnosed AL amyloidosis}

In the first-line setting, IV daratumumab was first administered in two patients with advanced cardiac involvement and they both achieved complete remission without serious adverse events. ${ }^{47}$ The phase III ANDROMEDA study demonstrated that the addition of SC daratumumab to standard CyBorD improved haematological and organ responses. ${ }^{6}$ Patients were randomized to receive either CyBorD alone for six cycles, or CyBorD plus daratumumab (DARA-CyBorD) for six cycles followed by maintenance therapy with monthly daratumumab for up to 24 cycles. ${ }^{6.48}$ Eligible patients had to have histopathological diagnosis of $\mathrm{AL}$ amyloidosis (based on immunohistochemistry or electron microscopy) with measurable disease and at least one involved organ according to International Society of Amyloidosis (ISA) criteria.99 Adequate organ function (estimated glomerular filtration rate of at least $20 \mathrm{~mL} /$ $\mathrm{min} / 1.73 \mathrm{~m}^{2}$ ) was required, and patients were not eligible if they were Mayo stage IIIB (NT-proBNP level $>8,500 \mathrm{ng} / \mathrm{L}$ ) or they had other severe cardiovascular diseases.

The primary endpoint of the study was overall haematological $C R$ rate. As a human IgGк MoAb, daratumumab is detected by serum immunofixation electrophoresis, making CR assessment according to ISA 
criteria challenging..$^{50}$ Thus, in ANDROMEDA, CR was defined as negative serum and urine immunofixation with an involved free light chain (iFLC) within normal limits but without the requirement of a normal FLC ratio. The study also established deep or stringent CRs (dFLC $<10 \mathrm{mg} / \mathrm{L}$ and iFLC $<20 \mathrm{mg} / \mathrm{L}$ ). Patients who met CR criteria were evaluated for MRD. Secondary endpoints of the study included major organ deteriorationprogression-free survival (MOD-PFS, a new composite endpoint that accounted for death, advanced cardiac or renal failure or haematological progression), organ response rate, PFS, OS, improvement in fatigue, 6-month haematological CR rate, at least haematological VGPR rate, time to and duration of haematological CR, and time to next treatment.

In the safety run-in analysis, 28 patients were evaluated for potential side effects. ${ }^{6}$ The most frequent all-grade adverse events were diarrhoea (67.9\%), fatigue (53.6\%) and peripheral oedema (50\%), and the most frequent grade $3 / 4$ events were fatigue (21.4\%), lymphopaenia (17.9\%) and anaemia (14.3\%), all of which were consistent with the toxicity profile of both SC daratumumab and CyBorD. Injection-related reactions occurred in one patient on the first and second administration, and were grade 1.

In the first interim analysis of the ANDROMEDA study, the median followup was 11.4 months. ${ }^{48}$ Overall, 388 patients were randomized (195 to the DARA-CyBorD group and 193 to the CyBorD-only control group), and haematological CR rate was $53.3 \%$ in the DARA-CyBorD group versus $18.1 \%$ in the CyBorD group (relative risk ratio $2.9,95 \%$ confidence interval $[\mathrm{Cl}] 2.1-4.1 ; \mathrm{p}<0.001) .{ }^{48}$ At the 6-month landmark analysis, haematological $\mathrm{CR}$ rate was $49.7 \%$ in the DARA-CyBorD group versus $14 \%$ in the CyBorD group. In the DARA-CyBorD group, median time to first response (at least partial response) was 9 days and median time to VGPR was 19 days. ${ }^{6}$ Importantly, the median time to haematological CR was 60 days in the DARA-CyBorD group versus 85 days in the CyBorD group. The rate of VGPR or better was $78.5 \%$ and $49.2 \%$ respectively $(p<0.0001)$, while deep haematological responses with iFLC $\leq 20 \mathrm{mg} / \mathrm{L}$ or $\mathrm{dFLC}$ levels $<10 \mathrm{mg} / \mathrm{L}$ more frequently occurred with the daratumumab combination treatment $(70.8 \%$ versus $20.2 \%$ and $64.1 \%$ versus $30.6 \%$, respectively). Independently of the criteria used, there was a substantial benefit in terms of deep haematological responses with the addition of daratumumab. In an updated analysis that was presented later, ${ }^{51}$ the median follow-up was 20.3 months, and haematological CR rates were significantly higher in the DARA-CyBorD group (59\% versus 19\%; odds ratio $5.9,95 \% \mathrm{Cl} 3.7-9.4 ; \mathrm{p}<0.0001$ )

Improvement of organ function is also a major goal of AL amyloidosis therapy. Patients who attain deep haematological responses are more likely to obtain an organ response and this can substantially improve their prognosis and survival.

In the interim analysis of ANDROMEDA, the addition of daratumumab to CyBorD was associated with higher frequencies of cardiac and renal responses. At the 6-month landmark analysis, the cardiac response rate was $41.5 \%$ in the DARA-CyBorD group and $22.2 \%$ in the CyBorD group $(p=0.0029),{ }^{48}$ and renal response rate was $53.0 \%$ and $23.9 \%$, respectively $(p<0.0001)$. Organ response rates were further enhanced at the 12 -month landmark analysis (cardiac response $57 \%$ versus $28 \%$; renal response $57 \%$ versus $27 \%)^{5}$

Regarding secondary endpoints, the MOD-PFS significantly favoured the DARA-CyBorD group (hazard ratio 0.58, 95\% Cl 0.36-0.93; $\mathrm{p}=0.0211$ ). ${ }^{48}$ The median time to next treatment in the DARA-CyBorD group was not reached, whereas in the CyBorD group it was 10.4 months. A total of 19 patients (9.8\%) in the DARA-CyBorD group and 79 patients (42.0\%) in the CyBorD group received subsequent therapy.

Early deaths ( $<60$ days from initiation of treatment) occurred in 13 patients in each group and a total of 56 patients died (27 in the DARACyBorD group and 29 in the CyBorD group). Overall death was considered to be related to adverse events in 23 patients $(11.9 \%)$ in the DARACyBorD group and in 14 patients (7.4\%) in the CyBorD group. Although not statistically significant, this may be attributed to the increased risk of infections and the immunoparesis related to daratumumab in patients with cardiac involvement. Death caused by disease progression was observed in $1.0 \%$ of patients in the DARA-CyBorD group versus $4.8 \%$ in the CyBorD group. Overall mortality and adverse events related to death were mostly reported in patients with advanced cardiac involvement.

The use of SC daratumumab saves considerable time and resources, and in the ANDROMEDA trial SC daratumumab was also correlated with fewer systemic administration-related reactions (in 14 patients [7.3\%]), all of which were mild (grade 1 or 2), and most of them (85.7\%) occurred during the first administration. Local injection-related reactions were also observed in 54 patients (28.0\%, versus 45 patients [23.9\%]), with 21 patients (10.9\%) in the DARA-CyBorD group experiencing injectionrelated reactions with daratumumab. Longer follow-up will be needed to assess the role of daratumumab maintenance in the course of the disease.

\section{Future perspectives}

The combination of bortezomib with cyclophosphamide and dexamethasone (CyBorD) results in VGPR/CR rates of $43 \%$, with organ responses in up to $25 \%$ of patients, and was considered the mainstay of $A L$ amyloidosis therapy until recently. The ANDROMEDA trial demonstrated that the addition of daratumumab to CyBorD, yields faster, deeper and more durable responses with limited toxicity. As a result, daratumumab has moved to the frontline of AL amyloidosis therapy and DARA-CyBorD has become the new standard of care.

A very appealing feature of daratumumab is its significant ability to induce deep haematological responses within the first month of its administration. Findings from the ANDROMEDA trial along with previously published data, 22,53 suggest that the achievement of stringent dFLC or iFLC responses, even without fulfilling ISA criteria for $\mathrm{CR}$, is associated with significantly improved survival and higher organ responses. As mentioned above, as a human IgGK moAb, daratumumab is detected by serum immunofixation electrophoresis, making $C R$ assessment challenging. ${ }^{50}$ Patients with measurable disease who achieve CR or very low FLCs levels and patients without measurable disease after the completion of therapy should be evaluated for MRD using next-generation flow cytometry or next-generation sequencing. Although the MRD data from the ANDROMEDA trial are not yet available, MRD-negative status in other series has been associated with very low probability of haematological relapse and with high rates of organ responses. ${ }^{54}$ These results highlight that MRD negativity should be the optimal goal of AL amyloidosis anticlonal therapy. Furthermore, as next-generation flow methods are increasingly available, a shift towards more individualized treatments is anticipated. Other antigens on the surface of plasma cells that have been targeted initially in myeloma but are also tested in AL amyloidosis, include CD20 surface molecule (targeted by rituximab), ${ }^{55}$ Signaling Lymphocyte Activation Marker Family member 7 (SLAMF7) receptor (targeted by elotuzumab) ${ }^{56}$ and B-cell maturation antigen (targeted by belantamab mafodotin antibody-drug conjugate)..$^{57}$ 
In AL amyloidosis, translocation $(11 ; 14)$ is the most common chromosomal alteration among patients (up to $50 \%) .{ }^{58}$ Carrying t(11;14) is an adverse prognostic factor, with inferior haematological responses in patients treated with bortezomib-based regimens (VGPR or better $52 \%$ versus 77\%; $p=0.004$ ) and with shorter survival (median OS 15 versus 27 months; $p=0.05) .12,59$ In the ANDROMEDA trial, haematological CR rates were independent of the presence of $\mathrm{t}(11 ; 14)(55 \% \text { versus } 52 \%)^{48}$ in the DARA-CyBorD group. In fact, the addition of daratumumab may overcome the adverse impact of $t(11 ; 14)$, rendering daratumumab a promising tool for this subgroup of patients, either as monotherapy or in combinations. In addition, $t(11 ; 14)$ is associated with overexpression of anti-apoptotic factor $\mathrm{BCl}-2$, which is targeted by the $\mathrm{BCl}-2$ inhibitor, venetoclax..$^{60,61}$ However, based on data from myeloma patients, high mortality rates raise concerns about safety in this frail population; some case reports have implied that the addition of venetoclax to daratumumab could enhance the outcome in patients with relapsed/ refractory AL amyloidosis. ${ }^{60}$

A very high-risk cohort of patients includes Mayo stage IIIB (NTproBNP levels $>8,500 \mathrm{pg} / \mathrm{mL}$ ) and patients with extremely low systolic blood pressure $\left(<100 \mathrm{mmHg}\right.$ ), with median survival of 3-6 months. ${ }^{3.62}$ The early eradication of the toxic FLCs and the underlying plasma cell clone is crucial. However, standard treatment with CyBorD is ill-tolerated. Daratumumab, on the other hand, is a regimen with manageable adverse events, no cardiotoxicity, and the introduction of SC administration significantly reduces the risk of volume overload. Given the high rates of substantial deep responses and the high rates of cardiac responses in relapsed AL but also in the ANDROMEDA study, daratumumab has emerged as an attractive option for high-risk patients. An ongoing phase II trial is currently assessing the safety and efficacy of daratumumab monotherapy in previously treatment-naive stage IIIB AL amyloidosis patients. ${ }^{63}$

The rapid reduction of the toxic FLCs is also relevant for the restoration of kidney function in patients with renal amyloidosis. The renal damage often leads to end-stage renal disease; therefore, the use of daratumumab, which induces CRS and VGPRs in a short time, in combination with bortezomib, which has a protective effect on the kidney, ${ }_{1}^{64}$ offers a very promising novel option. Daratumumab-based regimens have also been considered for the treatment of the rare entities of monoclonal gammopathies of renal significance, with great efficacy and safety in a small cohort of 25 patients. ${ }^{65}$ Although monoclonal antibodies are safe for the treatment of patients with renal dysfunction, in a series of 168 patients with AL amyloidosis treated with daratumumab-based regimens, nephrotic-range proteinuria was an adverse factor for haematological event-free survival. ${ }^{43}$ This may be attributed to the impaired glomerular function in patients with severe non-selective proteinuria and to urinary excretion of monoclonal antibodies. ${ }^{66}$

Besides cardiac and renal amyloidosis, there is also concern for the management of patients with mild-to-severe peripheral nerve involvement. Owing to the neurotoxicity of bortezomib and fears about exacerbation of neuropathy symptoms, daratumumab even as monotherapy could be the optimal choice. ${ }^{67}$
The advances in the field of AL amyloidosis will focus on the development of treatments that target the amyloid deposits because they could improve organ function. Phase I/II studies of CAEL-101, an IgG1 MoAb targeting light chain amyloid fibrils, demonstrated that it is safe and results in significant organ responses.68,69 A randomized phase III study in patients with advanced cardiac involvement is ongoing and currently recruiting patients..$^{70,71}$ The combination of anticlonal therapies that yield rapid and deep responses, such as daratumumab, with an effective amyloid targeting regimen that reduces amyloid load could change the natural history of the disease.

High-dose melphalan followed by ASCT is an effective treatment for appropriately selected AL amyloidosis patients, with haematological responses of $84 \%$ (CR 39\% and VGPR 33\%) and organ responses of $60 \%$, which may last for more than 15 years when performed in experienced centres. ${ }^{72,73}$ Features that support the use of ASCT in AL amyloidosis management include the small burden of clonal cells, the low proliferative index and the absence of high-risk cytogenetics abnormalities. ${ }^{72}$ However, treatment-related mortality rates, which may be as high as 20\%,74 and the introduction of novel immunotherapies make its role debatable. In fact, the attainment of deep haematological and organ responses with the use of daratumumab-based regimens as a first-line agent (CR 53\%, cardiac response $42 \%$ and renal response $53 \%$ ), together with its favourable safety profile, eliminate the need for ASCT in AL amyloidosis, even for transplant-eligible patients. Depending on the depth of haematological response and the achievement of organ response post-daratumumab, ASCT could be considered for those patients with residual disease or persistent organ dysfunction. Longer follow-up of the ANDROMEDA study will further elucidate the role of daratumumab maintenance therapy as a more appropriate chemotherapy-free strategy, compared with ASCT, for patients who have not achieved complete eradication of clonal cells.

In the setting of relapse, the establishment of DARA-CyBorD as the mainstay of first-line treatment raises new questions about the management of relapsed or refractory AL amyloidosis patients. Today, many patients are still naïve to daratumumab. Thus, single-agent therapy or combination with low-dose lenalidomide should be considered. Overall, large prospective clinical trials with modern regimens and novel combinations are needed to address these new challenges. The management strategy should always be tailored according to risk stratification systems, organ involvement, clonal cell biology and haematological response.

\section{Conclusions}

The overexpression of CD38 surface molecule has provided the rationale for using daratumumab in the treatment of patients with AL amyloidosis. The results of the ANDROMEDA study show that deep and durable haematological and organ responses are feasible with the addition of daratumumab to CyBorD and this led to the first approved treatment for the disease. Early diagnosis and immediate initiation of therapy are still the mainstay of AL amyloidosis management. The high efficacy and tolerability of daratumumab, even in high-risk populations, highlight a promising new era for the treatment of AL amyloidosis. $\square$
1. Palladini $\mathrm{G}$, Merlini $\mathrm{G}$. What is new in diagnosis and management of light chain amyloidosis? Blood. 2016;128:159-68

2. Dispenzieri A, Gertz MA, Kyle RA, et al. Serum cardiac troponins and $\mathrm{N}$-terminal pro-brain natriuretic peptide: a staging system for primary systemic amyloidosis. J Clin Oncol. 2004;22:3751-7.

3. Kumar S, Dispenzieri A, Lacy MQ, et al. Revised prognostic staging system for light chain amyloidosis incorporating cardiac biomarkers and serum free light chain measurements. J Clin oncol. 2012;30:989-95.
4. Muchtar E, Gertz MA, Kumar SK, et al. Improved outcomes for newly diagnosed AL amyloidosis between 2000 and 2014: cracking the glass ceiling of early death. Blood. 2014: cracking the

Kaufman GP, Dispenzieri A, Gertz MA, et al. Kinetics of Kaufman GP, Dispenzleri A, Gertz MA, et al. Kinetics of
organ response and survival following normalization of the serum free light chain ratio in AL amyloidosis. Am J Hemato 2015;90:181-6

6. Palladini G, Kastritis E, Maurer MS, et al. Daratumumab plus CyBorD for patients with newly diagnosed AL amyloidosis: safety run-in results of ANDROMEDA. Blood. 2020;136:71-80 US Food and Drug Administration. FDA grants accelerated approval to Darzalex Faspro for newly diagnosed light chain amyloidosis. Available at: https://www.fda.gov/drugs/drugamyloidosis. Avallable at: https://Www.fda.gov/drugs/drugapprovals-and-databases/fda-grants-accelerated-approval-
darzalex-faspro-newly-diagnosed-light-chain-amyloidosis darzalex-faspro-newly-diagnos

8. Gertz MA, Kyle RA, Greipp PR. The plasma cell labeling index: a valuable tool in primary systemic amyloidosis. Blood. 1989;74:1108-11. 
9. Kourelis TV, Kumar SK, Gertz MA, et al. Coexistent multiple myeloma or increased bone marrow plasma cells define equally high-risk populations in patients with immunoglobulin light chain amyloidosis. I Clin Oncol. 2013:31:4319-24.

10. Oliva $L$, Orfanelli $U$, Resnati $M$, et al. The amyloidogenic light chain is a stressor that sensitizes plasma cells to proteasome inhibitor toxicity. Blood. 2017;129:2132-42

11. Boyle EM, Ashby C, Wardell CP, et al. The genomic landscape of plasma cells in systemic light chain amyloidosis. Blood. 2018;132:2775-7.

12. Bochtler T, Hegenbart $\mathrm{U}$, Kunz $\mathrm{C}$, et al. Translocation $\mathrm{t}(11 ; 14)$ is associated with adverse outcome in patients with newly diagnosed AL amyloidosis when treated with bortezomibbased regimens. J Clin Oncol. 2015;33:1371-8.

13. Matsuda $\mathrm{M}$, Gono $\mathrm{T}$, Shimojima $\mathrm{Y}$, et al. Phenotypic analysis of plasma cells in bone marrow using flow cytometry in $\mathrm{AL}$ amyloidosis. Amyloid. 2003;10:110-16.

14. Seckinger $A$, Hillengass J, Emde $M$, et al. CD38 as immunotherapeutic target in light chain amyloidosis and multiple myeloma-association with molecular entities, risk, survival, and mechanisms of upfront resistance. Front Immunol. 2018;9:1676.

15. Deaglio S, Morra M, Mallone R, et al. Human CD38 (ADP-ribosyl cyclase) is a counter-receptor of CD31, an Ig superfamily member. J Immunol. 1998;160:395-402.

16. Ferrero E, Malavasi F. Human $C D 38$, a leukocyte receptor and ectoenzyme, is a member of a novel eukaryotic gene family of nicotinamide adenine dinucleotide+-converting enzymes: extensive structural homology with the genes for murine bone marrow stromal cell antigen 1 and aplysian ADP-ribosy cyclase. J Immunol. 1997;159:3858-65.

17. Flores-Borja F, Bosma A, Ng D, et al. CD19+CD24hiCD38hi B cells maintain regulatory T cells while limiting TH1 and TH17 differentiation. Sci Trans/ Med. 2013:5:173ra123.

18. Krejcik J, Casneuf T, Nijhof IS, et al. Daratumumab depletes CD38+ immune regulatory cells, promotes T-cell expansion, and skews T-cell repertoire in multiple myeloma. Blood. and skews T-cell repr

19. Zambello R, Barilà $G$, Manni $S$, et al. NK cells and CD38: implication for (immuno)therapy in plasma cell dyscrasias Cells. 2020;9:768.

20. de Weers M, Tai Y-T, van der Veer MS, et al. Daratumumab, a novel therapeutic human CD38 monoclonal antibody, induces killing of multiple myeloma and other hematological tumors. Immunol. 2011;186:1840-8.

21. van de Donk N, Richardson PG, Malavasi F. CD38 antibodies in multiple myeloma: back to the future Blood 2018:131:13-29.

22. Overdijk MB, Verploegen S, Bögels M, et al Antibody-mediated phagocytosis contributes to the anti-tumor activity of the therapeutic antibody daratumumab in lymphoma and multiple myeloma. MAbs. 2015;7:311-21.

23. Overdijk MB, Jansen $\mathrm{JH}$, Nederend $\mathrm{M}$, et al. The therapeutic CD38 monoclonal antibody daratumumab induces programmed cell death via Fcy receptor-mediated crossLinking. J Immunol. 2016;197:807-13.

24. van de Donk NWCJ. Immunomodulatory effects of CD38targeting antibodies. Immunol Lett. 2018;199:16-22.

25. Adams HC, 3rd, Stevenaert F, Krejcik J, et al. High-parameter mass cytometry evaluation of relapsed/refractory multiple myeloma patients treated with daratumumab demonstrates immune modulation as a novel mechanism of action. Cytometry A. 2019:95:279-89.

26. Lammerts van Bueren J, Jakobs D, Kaldenhoven N, et al. Direct in vitro comparison of daratumumab with surrogate analogs of CD38 antibodies MOR03087, SAR650984 and Ab79. Blood. 2014:124:3474-4.

27. Lonial S, Weiss BM, USmani SZ, et al. Daratumumab monotherapy in patients with treatment-refractory multiple myeloma (SIRIUS): an open-label, randomised, phase 2 trial. Lancet. 2016;387:1551-60.

28. Lokhorst HM, Plesner T, Laubach JP, et al. Targeting CD38 with daratumumab monotherapy in multiple myeloma. N Eng/ Med. 2015;373:1207-19.

29. Dimopoulos MA, Oriol A, Nahi H, et al. Daratumumab, lenalidomide, and dexamethasone for multiple myeloma. N Eng/ J Med. 2016;375:1319-31.

30. Palumbo A, Chanan-Khan A, Weisel K, et al. Daratumumab, bortezomib, and dexamethasone for multiple myeloma. N Eng J Med. 2016:375:754-66.

31. Facon T, Kumar S, Plesner T, et al. Daratumumab plus Ienalidomide and dexamethasone for untreated myeloma. N Eng/ J Med. 2019;380:2104-15.

32. Mateos MV Dimopoulos MA, Cavo $M$, et al. Daratumumab plus bortezomib, melphalan, and prednisone for untreated myeloma. N Eng/ J Med. 2018;378:518-28.

33. Moreau $\mathrm{P}$, Attal M, Hulin $\mathrm{C}$, et al Bortezomib, thalidomide, and dexamethasone with or without daratumumab before and after autologous stem-cell transplantation for newly diagnosed multiple myeloma (CASSIOPEIA): a randomised, open-label, phase 3 study. Lancet. 2019:394:29-38.

34. Voorhees PM, Kaufman JL, Laubach J, et al. Daratumumab, lenalidomide, bortezomib, and dexamethasone for transplanteligible newly diagnosed multiple myeloma: the GRIFFIN trial. Blood. 2020;136:936-45.

35. Bahlis NJ, Dimopoulos MA, White DJ, et al. Daratumumab plus lenalidomide and dexamethasone in relapsed/ refractory multiple myeloma: extended follow-up of POLLUX, a randomized, open-label, phase 3 study. Leukemia. 2020;34:1875-84

36. Spencer A, Lentzsch S, Weisel K, et al. Daratumumab plus bortezomib and dexamethasone versus bortezomib and dexamethasone in relapsed or refractory multiple myeloma: updated analysis of CASTOR. Haematologica. 2018;103:207987.

37. Sher T, Fenton B, Akhtar A, et al. First report of safety and efficacy of daratumumab in 2 cases of advanced immunoglobulin light chain amyloidosis. Blood. 2016;128:1987-9

38. Kaufman GP, Schrier SL, Lafayette RA, et al. Daratumumab yields rapid and deep hematologic responses in patients with heavily pretreated AL amyloidosis. Blood. 2017;130:900-2.

39. Khouri J, Kin A, Thapa B, et al. Daratumumab proves safe and highly effective in AL amyloidosis. Br J Haematol. 2019;185:342-4

40. Sanchorawala V, Sarosiek S, Schulman A, et al. Safety, tolerability, and response rates of daratumumab in relapsed AL amyloidosis: results of a phase 2 study. Blood. 2020;135:1541-7.

41. Roussel $\mathrm{M}$, Merlini $\mathrm{G}$, Chevret $\mathrm{S}$, et al. A prospective phase 2 trial of daratumumab in patients with previously treated systemic light-chain amyloidosis. Blood 2020;135:1531-40.

42. Chung A, Kaufman GP, Sidana S, et al. Organ responses with daratumumab therapy in previously treated AL amyloidosis Blood Adv. 2020;4:458-66.

43. Kimmich CR, Terzer T, Benner A, et al. Daratumumab for systemic AL amyloidosis: prognostic factors and adverse outcome with nephrotic-range albuminuria. Blood. 2020;135:1517-30

44. Milani P, Fazio F, Basset M, et al. High rate of profound clona and renal responses with daratumumab treatment in heavily pre-treated patients with light chain (AL) amyloidosis and high bone marrow plasma cell infiltrate. Am J Hemato. 2020;95:900-5.

45. Abeykoon JP, Zanwar S, Dispenzieri A, et al. Daratumumabbased therapy in patients with heavily-pretreated $\mathrm{AL}$ amyloidosis. Leukemia. 2019:33:531-6.

46. ClinicalTrials.gov. A Study to Evaluate the Efficacy and Safety of Daratumumab in Combination with Cyclophosphamide, Bortezomib and Dexamethasone (CyBorD) Compared to CyBorD Alone in Newly Diagnosed Systemic Amyloid Light-chain (AL) Amyloidosis. ClinicalTrials.gov Identifier: NCT03201965. Available at: https://www.clinicaltrials.gov/ct2/ show/NCT03201965 (accessed 26 November 2021).

47. Hossein Taghizadeh M, Thomas R, Franz D, et al. Daratumuma - a safe first-line treatment of cardiac AL amyloidosis in heavily compromised patients. The XVIth International Symposium on Amyloidosis; Kumamoto, Japan, 26-29 March, 2018.

48. Kastritis E, Palladini G, Minnema MC, et al. Daratumumab-based treatment for immunoglobulin light-chain amyloidosis. N Eng/ J Med. 2021;385:46-58.

49. Gertz MA, Comenzo R, Falk RH, et al. Definition of organ involvement and treatment response in immunoglobulin light chain amyloidosis (AL): a consensus opinion from the 10th International Symposium on Amyloid and Amyloidosis, Tours, France, 18-22 April 2004. Am J Hematol. 2005;79(4):319-28.

50. McCudden C, Axel AE, Slaets D, et al. Monitoring multiple myeloma patients treated with daratumumab: teasing out monoclonal antibody interference. Clin Chem Lab Med. 2016;54:1095-104.

51. Kastritis E, Sanchorawala V, Merlini G, et al. Subcutaneous daratumumab + bortezomib, cyclophosphamide, and dexamethasone (VCd) in patients with newly diagnosed light chain (AL) amyloidosis: updated results from the phase 3 ANDROMEDA study. J Clin Oncol. 2021;39(Suppl. 15):8003.

52. Muchtar E, Dispenzieri A, Leung N, et al. Optimizing deep response assessment for $\mathrm{AL}$ amyloidosis using involved free light chain level at end of therapy: failure of the serum free light chain ratio. Leukemia. 2019:33:527-31.

53. Manwani $\mathrm{R}$, Cohen $\mathrm{O}$, Sharpley $\mathrm{F}$, et al. A prospective observational study of 915 patients with systemic AL amyloidosis treated with upfront bortezomib. Blood. 2019;134:2271-80

54. Kastritis E, Kostopoulos IV, Theodorakakou F, et al. Next generation flow cytometry for MRD detection in patients with AL amyloidosis Amyloid. 2021:28:19-23.

55. Manwani R, Sachchithanantham S, Mahmood S, et al. Treatment of IgM-associated immunoglobulin light-chain amyloidosis with rituximab-bendamustine. Blood. 2018;132:761-4.

56. Iqbal SM, Stecklein K, Sarow J, et al. Elotuzumab in combination with lenalidomide and dexamethasone for treatment-resistant immunoglobulin light chain amyloidosis with multiple myeloma. Clin Lymphoma Myeloma Leuk. 2019;19:e33-e36.

57. ClinicalTrials.gov. A Study of Belantamab Mafodotin in Patients with Relapsed or Refractory AL Amyloidosis (EMN27). ClinicalTrials.gov Identifier: NCT04617925. Available at: https:// clinicaltrials.gov/ct2/show/NCT04617925 (accessed 26 November 2021).

58. Hayman SR, Bailey RJ, Jalal SM, et al. Translocations involving the immunoglobulin heavy-chain locus are possible early genetic events in patients with primary systemic amyloidosis. genetic events in patients
Blood. 2001;98:2266-8.

59. Muchtar E, Dispenzieri A, Kumar SK, et al. Interphase fluorescence in situ hybridization in untreated AL amyloidosis has an independent prognostic impact by abnormality type and treatment category. Leukemia. 2017;31:1562-9.

60. Yip PL, Lau JSM, Lam CP. Venetoclax monotherapy induced rapid and sustained response in a frail patient with refractory AL amyloidosis: less is more? Int J Hematol. 2020;112:234-7.

61. Leung N, Thomé SD, Dispenzieri A. Venetoclax induced a complete response in a patient with immunoglobulin light chain amyloidosis plateaued on cyclophosphamide, bortezomib and dexamethasone. Haematologica. 2018; 103:e135-e137.

62. Wechalekar AD, Schonland SO, Kastritis E, et al. A European collaborative study of treatment outcomes in 346 patients with cardiac stage III AL amyloidosis. Blood. 2013:121:3420-7.

63. ClinicalTrials.gov. A Study of Daratumumab Monotherapy in Previously Untreated Patients with Stage 3B Light Chain (AL) Amyloidosis. ClinicalTrials.gov Identifier: NCT04131309. Available at: https://clinicaltrials.gov/ct2/show/NCT04131309 (accessed 26 November 2021

64. Chanan-Khan AA, Kaufman JL, Mehta J, et al. Activity and safety of bortezomib in multiple myeloma patients with advanced renal failure: a multicenter retrospective study. Blood. 2007;109:2604-6.

65. Kastritis E, Theodorakakou F, Roussou M, et al. Daratumumabbased therapy for patients with monoclonal gammopathy of renal significance. Br J Haematol. 2021;193:113-18.

66. Jacobs R, Langer-Jacobus T, Duong M, et al. Detection and quantification of rituximab in the human urine. I Immunol Methods. 2017:451:118-21.

67. Deshpande S, Gertz MA, Dispenzieri A, et al. Daratumumab as successful initial therapy for AL amyloidosis with nerve involvement. Leuk Lymphoma. 2020;61:1752-5.

68. Edwards CV, Gould J, Langer AL, et al. Interim analysis of the phase $1 \mathrm{a} / \mathrm{b}$ study of chimeric fibril-reactive monoclonal antibody 11-1F4 in patients with AL amyloidosis. Amyloid. 2017;24:58-9

69. Khouri J, Anwer F, Samaras CJ, et al. Safety, tolerability and efficacy of Cael-101 in AL amyloidosis patients treated on a phase 2, open-label, dose selection study to evaluate the safety and tolerability of Cael-101 in patients with AL amyloidosis. Blood. 2020;136 (Suppl. 1):21.

70. ClinicalTrials.gov. A Study to Evaluate the Effectiveness and Safety of CAEL-101 in Patients with Mayo Stage IIla AL Amyloidosis. ClinicalTrials.gov Identifier: NCT04512235. Available at: https://clinicaltrials.gov/ct2/show/NCT04512235 (accessed 26 November 2021).

71. ClinicalTrials.gov. A Study to Evaluate the Effectiveness and Safety of CAEL-101 in Patients with Mayo Stage IIIb AL Amyloidosis. ClinicalTrials.gov Identifier: NCT04504825. Available at: https://clinicaltrials.gov/ct2/show/NCT04504825 (accessed 26 November 2021.

72. Sidiqi MH, Aljama MA, Buadi FK, et al. Stem cell transplantation for light chain amyloidosis: decreased early mortality over time. J Clin Oncol. 2018;36:1323-9.

73. Sharpley FA, Petrie A, Mahmood S, et al. A 24-year experience of autologous stem cell transplantation for light chain amyloidosis patients in the United Kingdom. Br $\perp$ Haemato 2019;187:642-52.

74. D'Souza A, Dispenzieri A, Wirk B, et al. Improved outcomes after autologous hematopoietic cell transplantation for light chain amyloidosis: a Center for International Blood and Marrow Transplant Research Study. J Clin Oncol. 2015;33:3741-9. 